\title{
Dimensões psíquicas do emagrecimento: por uma compreensão psicanalítica da compulsão alimentar
}

\section{Psychic dimensions of weight loss: for a psychoanalytic understanding of binge eating disorder}

\author{
Juliana Ferreira Santos Farah*1 \\ Pablo Castanho*2
}

Com este artigo propomos uma pesquisa teórico-clínica com o objetivo de apresentar um entendimento psicanalítico da compulsão alimentar. Para tanto, apresentamos uma revisão crítica da literatura articulada com vinhetas de atendimentos e depoimentos públicos de pacientes. Concluiremos sublinhando o potencial de uma abordagem psicanalítica da compulsão alimentar em destacar a existência de riscos de desorganização psíquica de alguns pacientes submetidos a dietas de alta restrição calórica e seu potencial para lidar com os ciclos viciosos de perda e posterior ganho de peso.

Palavras-chave: Distúrbios do ato de comer, obesidade, psicanálise, comportamento compulsivo

${ }^{* 1}$ Mestranda de Psicologia na Universidade de São Paulo - USP (São Paulo, SP, Br).

*2 Universidade de São Paulo - USP (São Paulo, SP, Br). 


\section{Introdução}

Este artigo deriva de questionamentos surgidos da experiência profissional de um dos seus autores em uma clínica especializada em tratamento de sobrepeso e obesidade na qual, além de uma dieta prescrita por uma nutricionista, aos pacientes era indicada psicoterapia em grupo. O tratamento proposto por essa clínica parte da premissa de que a obesidade está diretamente ligada às adicções e tem como principal referência teórica as ideias propostas e discutidas por Zukerfeld (1979; 1996) e Ravenna (2006). Nesta perspectiva, uma abstinência do objeto-droga, ou seja, da comida, faz-se necessária para que haja tratamento, mas sozinha não é suficiente, por isso a indicação de psicoterapia de grupo. Embora esses autores não sejam psicanalistas e, consequentemente, a psicoterapia oferecida pela clínica também não priorize a abordagem psicanalítica, alguns dos profissionais contratados para conduzir tais grupos o são, como é o caso da primeira autora deste texto. Nestas situações, a condução do grupo se pautava por princípios psicanalíticos, como o convite à livre associação e a escuta flutuante, aproximando-se, de modo geral, de uma concepção psicanalítica do trabalho com grupos em instituição (Castanho, 2014).

Tal experiência suscitou questões para uma compreensão psicanalítica dos fenômenos ali vivenciados. Em especial, uma cena recorrente se apresenta para a análise. Após um período de maior dificuldade inicial - uma fase de "obedecer regras" e viver a abstinência física, seguindo a dieta indicada pela clínica —, a comida deixa de ocupar um lugar central 


\section{ARTIGOS}

no discurso dos pacientes sobre sua vida, abrindo espaço para o aparecimento de outras questões. Por vezes, ocorre apenas a substituição da centralidade da comida por algum outro objeto, como, por exemplo, a compra. Não raro, entretanto, os pacientes relatam a percepção de relações que não fazem sentido em suas vidas, raivas silenciadas, afetos calados.

Neste contexto, o objetivo deste trabalho é apresentar um entendimento psicanalítico da compulsão alimentar. Para tanto, partiremos de uma breve revisão da literatura sobre a problemática do emagrecimento, seguida de uma problematização da questão da compulsão alimentar e sua relação com a adicção em psicanálise. Utilizaremos algumas vinhetas de atendimentos e depoimentos para articular nossas reflexões. Concluiremos sublinhando o potencial de uma abordagem psicanalítica da compulsão alimentar em destacar a existência de riscos de desorganização psíquica de alguns pacientes submetidos a dietas de alta restrição calórica e seu potencial para lidar com os ciclos viciosos de perda e posterior ganho de peso.

\section{Sobre a obesidade na literatura científica}

A Organização Mundial de Saúde (OMS) tem alertado há alguns anos a respeito do aumento significativo da obesidade em vários países do mundo. Em estudo recente, essa entidade mostra que a obesidade mundial mais do que dobrou desde 1980, atingindo, em 2014, 13\% da população (World Health Organization, 2015). Essa epidemia estaria ligada fundamentalmente ao aumento da oferta de alimentos com alto índice calórico, ao aumento da urbanização, à dependência de veículos motorizados para a locomoção e aos trabalhos sedentários. Com a intenção de contê-la, a OMS divulga dados e orientações para prevenção e tratamento da obesidade, que priorizam os aspectos educativos com relação à alimentação e à prática de atividades físicas. Não aparecem, nessa publicação, referências a outros fatores etiológicos da obesidade, tais como os genéticos, os neurológicos e os psicológicos.

$\mathrm{Na}$ revisão bibliográfica feita por Tavares, Nunes e Santos (2010), podemos ver que não é só a OMS que coloca sobre os fatores psicológicos um enfoque menor do que consideramos devido. De acordo com os autores, os principais tratamentos utilizados são dieta, atividade física e prescrição de fármacos, mas isso continua produzindo, porém, resultados insatisfatórios, já que $95 \%$ dos pacientes com obesidade de grau III recuperam seu peso 
inicial em até dois anos após o fim do tratamento. Devido à dificuldade de abordagem clínica desses pacientes, a indicação de cirurgia bariátrica tem se tornado cada vez mais frequente. Os autores dessa revisão são fisioterapeutas e concluem o artigo, depois de examinarem a qualidade de vida dos obesos, apontando para a importância "da assistência multidisciplinar, buscando a melhora física, psíquica e social, visando a aprimorar e melhorar a qualidade da assistência oferecida" (Tavares, Nunes e Santos 2010, p. 365).

Magdaleno, Chaim e Turato (2009) alertam para o grande número de pacientes submetidos à cirurgia bariátrica que apresentam complicações psicológicas e psiquiátricas no pós-operatório. Ressaltam a importância de uma avaliação psicodinâmica dos pacientes que buscam a cirurgia bariátrica, uma vez que isso pode orientar o acompanhamento pós-operatório ou mesmo excluir o procedimento cirúrgico das recomendações de tratamento. A partir da observação de um grupo terapêutico de pacientes submetidos à cirurgia bariátrica, os autores relatam as dificuldades enfrentadas no pós-cirúrgico: a ideia de que a cirurgia, junto com a redução de peso, levaria embora magicamente todos os problemas é frustrada, abrindo espaço para uma angústia antes tamponada.

Zukerfeld (1979) aponta que durante muito tempo a obesidade foi uma enfermidade restrita ao campo da endocrinologia e, mesmo nessa área, desvalorizada como objeto de investigação em comparação com outras patologias. Segundo ele, foi a partir dos trabalhos de Bruch, do final da década de 1930, início da década de 1940, que a obesidade passou a ser considerada uma patologia psicossomática. Destacamos também o trabalho de Wulff, de 1932, que, dentro do campo psicanalítico, relacionou a compulsão alimentar à adicção (Wulff, 1932/2003).

No entanto, o que observamos desde então, é que dentre as modalidades de tratamento que enfocam os aspectos psicológicos da obesidade, são as terapias cognitivo-comportamentais que têm ganhado mais espaço. Mundim (1996) afirma que “(...) por não ser uma ferramenta de trabalho que se submete às estatísticas, toda forma de psicanálise parece ser rejeitada, negando ao médico um modelo teórico extremamente útil para compreender suas dificuldades e a dos obesos" (p. 22). De fato, nossa pesquisa dos termos "psicanálise" e "obesidade" — em todos os campos dos formulários de busca das bases de dados Pepsic e PsychoINFO (APA) - encontrou poucos artigos que tratam da obesidade a partir de uma leitura psicanalítica. Na brasileira Pepsic, encontramos 19 artigos. Na norte-americana APA (American Psychological Association) são 81 ocorrências para a busca dos termos 


\section{ARTIGOS}

psychoanalysis e obesity, enquanto para cognitive behavior therapy e obesity o número sobe para 680.

Dentro do campo psicanalítico, a associação psique e corpo não é novidade; é algo presente desde Freud. Nas palavras de Fernandes (2011), "a teoria freudiana permitiria colocar em evidência que o somático, isto é, o conjunto das funções orgânicas em movimento, habita um corpo que é também o lugar da realização de um desejo inconsciente" (p. 42).

Entretanto, se em Freud o corpo das psiconeuroses, isto é, o corpo da representação, foi privilegiado, na contemporaneidade temos observado uma proliferação de manifestações somáticas que não respondem a essa lógica. Ferraz (2011) retoma a presença das neuroses atuais na obra freudiana para associá-las a essas manifestações somáticas que, seja pela via do adoecimento, seja pela predominância da sua ação, não são frutos do recalcamento. $\mathrm{O}$ autor se pergunta por que as neuroses atuais teriam caído em esquecimento e afirma que

quando o corpo erógeno, este conceito genialmente descoberto a partir do estudo da histeria, ganhou a cena como local em que se processavam os sintomas psiconeuróticos, o corpo somático sofreu um apagão no pensamento psicanalítico. É assim que as funções remanescentes do corpo - aquelas ligadas a domínio somático ou ao registo da necessidade — foram deixadas de lado. (p. 242)

\section{Obesidade e compulsão alimentar — o que a psicanálise tem a dizer?}

Como foi dito anteriormente, dentro do campo psicanalítico há poucos artigos sobre a obesidade, e quando ampliamos a pesquisa para os transtornos alimentares, é sobre a anorexia que encontramos um número maior de publicações. Neste sentido, há também um número mais expressivo de artigos e capítulos de livros que tratam da compulsão alimentar presente na bulimia.

É importante ressaltar que a anorexia, a bulimia e a compulsão alimentar são diagnósticos psiquiátricos, descritos no DSM (Manual de Diagnóstico e Estatística das Perturbações Mentais), sendo que a compulsão alimentar foi inserida como apêndice na versão IV (em 1995), como transtorno da compulsão alimentar periódica — TCAP e incorporado formalmente em 2013 na versão 5, como transtorno da compulsão alimentar (Espíndola e Blay, 2006; American Psychiatric Association, 2014). 
Devemos nos perguntar se esses diagnósticos da psiquiatria fazem sentido enquanto entidades nosográficas na psicanálise. No nosso entendimento, não. Estamos de acordo com Kalil (2010) quando ela se refere aos episódios de compulsão alimentar como "manifestações sintomáticas de etiologia complexa e componentes adictivos" e afirma encontrar na clínica "a manifestação da compulsão alimentar nas mais diversas estruturas (neurose, psicose, perversão e psicossomática)" (p. 177). Isso implica que pensemos em uma clínica psicanalítica das compulsões alimentares — uma vez que não estamos falando de uma sintomatologia exclusiva de um único modo de subjetivação - , o que tem repercussões também nas formas de tratamento.

Ao se referir aos transtornos das condutas alimentares, Jeammet (2008) reforça a importância de não nos deixarmos enganar pela "aparente simplicidade ligada ao caráter muito estereotipado de sua expressão comportamental" (p. 30). O autor aponta como a evolução da psicanálise, especialmente nos últimos quarenta anos, contribuiu para uma leitura mais atenta à complexidade presente na clínica dos transtornos alimentares. Como vimos anteriormente, a ampliação da psicanálise para além do campo das neuroses clássicas permitiu que pudéssemos deslocar a ênfase "da análise dos conflitos ligados ao recalcamento para as particularidades da análise do narcisismo ou para as modalidades da relação de objeto" (Jeammet, 2008, p. 30).

Em um texto de 1979, Zukerfeld estuda as possíveis relações entre a esquizofrenia e a obesidade, indicando um desejo de iniciar uma discussão a respeito do tema até então pouco desenvolvido. Cita Bruch, que já na década de 1940 distinguiu um tipo específico de obesidade, denominada por ela de obesidade do desenvolvimento, que vai se desenvolvendo na infância, manifesta-se na adolescência e tem como uma das características uma significativa alteração da imagem corporal.

As pessoas com sobrepeso ou obesidade têm seus corpos biológicos claramente modificados, cobertos por gordura, podendo ou não ter consequências em órgãos internos e transtornos metabólicos. Mas o que acontece com a imagem corporal? Zukerfeld (1979) supõe que as alterações da imagem corporal do obeso sejam similares às que se dão na psicose. Citando Bruch, ele diz que a obesidade e o consumo excessivo de alimentos podem servir como uma defesa contra manifestações psíquicas mais graves, como uma depressão severa ou a esquizofrenia. Assim como na psicose, nesse tipo de obesidade há a evocação de processos primitivos e falta simbolização. 


\section{ARTIGOS}

Mas a evocação de processos primitivos e falta de simbolização também podem ser relacionadas à problemática da somatização em geral. Joyce McDougall (2000) afirma que:

O pensamento do psicótico pode ser concebido como uma "inflação delirante" do emprego da palavra com a finalidade de preencher os espaços de vazio aterrorizante (Montgrain, 1987), enquanto os processos de pensamento dos somatizantes procuram esvaziar a palavra de sua significação afetiva (McDougall, 1982a). Nos estados psicossomáticos, é o corpo que se comporta de maneira "delirante"; ele "hiperfunciona" ou inibe funções somáticas normais e o faz de modo insensato no plano fisiológico. O corpo enlouquece. (p. 22; itálicos nossos)

Nos grupos de psicoterapia da clínica alguns pacientes pareciam impermeáveis às interpretações e intervenções, mantendo o discurso centrado na comida ou em racionalizações e intelectualizações. Havia outros que, afastados do consumo excessivo da comida, relatavam uma angústia insuportável e rapidamente voltavam a comer, muitas vezes escondidos. O que é comum aos dois tipos de manifestação é o caráter insuportável da dieta restritiva e a impossibilidade da angústia emergente encontrar caminhos para tramitar, pelas palavras, no grupo de psicoterapia. Talvez, justamente porque estejamos confrontando aspectos insuficientemente simbolizados para que isso possa ocorrer.

Seguindo Klein (1946/1991), é na passagem para a posição depressiva que se inicia o processo de formação de símbolos, dando ao ego ferramentas para lidar com as ansiedades em relação a perda do objeto. Mas se o desenvolvimento na posição esquizoparanoide não se processou satisfatoriamente na infância, e o bebê não conseguiu lidar com os impactos das ansiedades depressivas, o ego é forçado a regredir à posição esquizoparanoide, mantendo e reforçando temores persecutórios precoces e fenômenos esquizoides.

Podemos pensar que alguns pacientes, como os citados acima, vivem uma impossibilidade de aceitar a perda do objeto, mantendo-se em relações esquizoides de objeto. Nesses casos, ao deixarem de comer compulsivamente, vivem um aumento significativo da ansiedade persecutória, antes suprimida com a ingestão excessiva de alimentos.

Há casos, como aponta Zukerfeld (1979), em relação aos quais não devemos supor que a perda de peso possa colocar o sujeito em uma situação depressiva, o que implicaria elaboração e reparação ou, inversamente, que quando passa por um processo psicanalítico ele possa alcançar a posição depressiva e, consequentemente, emagrecer. Ao contrário, como mencionado 
anteriormente, o que devemos reconhecer é que em alguns pacientes a restrição alimentar desvela angústias insuportáveis cujos efeitos desestruturantes sobre o psiquismo não devem ser ignorados.

Nesses casos, estamos certamente diante de uma relação adictiva com o alimento, que opera na tentativa de combater angústias. Mas esta não é a única modalidade de relação adictiva com os alimentos que podemos identificar. McDougall (1995) propõe que diferentes graus de angústia geram diferentes estados psíquicos e, portanto, diferentes soluções adictivas. Tais soluções adictivas podem estar ligadas a angústias psicóticas, tais como o medo da fragmentação corporal e psíquica, um terror global de encarar um vazio, no qual o próprio sentimento de identidade subjetiva é sentido como estando em perigo. Podem estar ligadas também a angústias que a autora denomina de "graves", frequentemente de natureza paranoide, ou de depressão. E, finalmente, a angústias neuróticas, ou seja, conflitos ligados às relações amorosas sexuais e ao prazer narcísico no trabalho e nas relações sociais (p. 202). Essa dimensão neurótica da relação adictiva com o objeto também encontramos em nossa clínica com pacientes submetidos a dietas de alta restrição calórica e o diagnóstico diferencial pode não ser evidente.

Portanto, o comer compulsivo presente em alguns casos de obesidade pode ser considerado um comportamento adictivo e pode se apresentar como tentativa de combater a angústia em diferentes modos de constituição subjetiva. McDougall (2000) aponta que a adicção "pode ser considerada como uma tentativa 'psicossomática' de superar a dor mental através do recurso a substâncias externas que tranquilizam o espírito e suprimem provisoriamente o conflito psíquico" (p. 22-23). A mesma autora (1983) sustenta que além da somatização, de alguns desvios sexuais e de algumas neuroses caracteriais, as adicções (por alimentos, tabaco, álcool, medicamentos, entre outras) podem ser chamados de atos-sintomas.

Atos-sintomas são definidos por McDougall (1983) como manifestação da fragilidade na elaboração psíquica e da falha na simbolização, compensadas por um agir compulsivo que procura reduzir a intensidade da dor psíquica. Segundo ela:

Todo ato-sintoma ocupa o lugar de um sonho nunca sonhado, de um drama em potencial, onde as personagens desempenham o papel de objetos parciais ou até são disfarçados de objetos-coisa, numa tentativa de imputar aos objetos substitutivos a função de um objeto simbólico que está ausente ou danificado no mundo psíquico (ex. os alimentos ou a droga que servem como resposta à depressão). (p. 134) 


\section{ARTIGOS}

Gurfinkel (2011) aponta, em seu livro Adicções, a correlação entre oralidade e adicções, de alguma forma já presente em "Três ensaios sobre a sexualidade" (1905), quando Freud propõe que, se há uma permanência da importância erógena da zona labial na vida adulta, pode haver o que o autor chama de "beijos perversos", consumo de bebidas e excesso no fumar. Gurfinkel destaca também a grande contribuição de Karl Abraham para a linha de pesquisa que associa organização oral da libido e adicções. A compulsão alimentar foi, inclusive, a primeira forma de adicção descrita por ele em seu artigo de 1916 sobre o tema (Gurfinkel, 2011).

Nessa perspectiva, diferente do que muitos pacientes pensam, comer tudo não é um indicador de liberdade. Ao contrário, a sensação de urgência em comer aponta para uma impossibilidade de escolha. Isso nos remete à etimologia da palavra adicção, que, como nos aponta Gurfinkel (2011), refere-se a alguém que se tornou escravo por conta de uma dívida e essa característica

implica uma importante inversão da relação sujeito-objeto, nos seguintes termos: aquele que era o sujeito que, no exercício de sua liberdade, escolhia usar o objeto segundo sua vontade e a serviço de seu desejo, se torna ele mesmo objeto de seu objeto, que ganha, por sua vez, o estatuto de dono e senhor da situação. (p. 51; itálico do autor)

O depoimento a seguir, retirado da página da internet de uma clínica de emagrecimento, ilustra a intensidade desta inversão vivida por C., uma moça de 30 anos:

Desejo, de coração, entrar numa padaria e não ter as pupilas dilatadas e os batimentos cardiacos acelerados. Isso AINDA não foi possivel, mas tenho curtido bastante ser alguém que faz escolhas saudáveis. ${ }^{1}$

A aproximação, do ponto de vista psicanalítico, entre compulsão alimentar e adicções, aponta para um outro fenômeno que também pode ser observado com frequência na clínica e ao qual já aludimos na introdução deste artigo: o deslocamento da compulsão por comida para um outro objeto-droga. Essa observação é reforçada pela literatura, como podemos ver em Marcelino e Patrício (2011, p. 4774), que em seu estudo sobre ex-obesos mórbidos evidenciaram que parte deles, após o emagrecimento, desenvolvem, entre outras coisas, dependência a álcool e outras drogas, compulsões por jogos, compras ou sexo.

${ }^{1} \mathrm{http}: / /$ maximoravenna.com.br/c-s-c-30-anos-sexo-feminino/\#more-1192 
$\begin{array}{llllllllllll}R & E & V & \text { I } & S & T & A\end{array}$

LATINOAMERICANA

DE PSICOPATOLOGIA

F U N D A M N T A L

Você tem fome de quê?

A gente não quer só comer

A gente quer comer

E quer fazer amor

A gente não quer só comer

A gente quer prazer

Prá aliviar a dor..

(Arnaldo Antunes, Sérgio Brito e Marcelo Fromer)

Como na música dos Titãs, o ato de comer nem sempre está associado apenas a uma necessidade fisiológica, mas pode ter uma função de "aliviar a dor". É o caso de Antonia (nome fictício), que durante um grupo de psicoterapia, ao escutar da terapeuta que aquilo que ela estava sentindo não era fome, ficou extremamente incomodada e reativa. Algumas semanas depois, Antonia pediu a palavra no grupo e contou que passou por uma tentativa de assalto à mão armada e que percebeu que isso a deixou extremamente faminta. Chegando em casa, diante da possibilidade de furar a dieta e acabar com aquela "fome", lembrou-se da fala da terapeuta e pensou: "acho que isso não 50 é fome...". Pela primeira vez em muitos anos, Antonia percebeu que diante de uma situação de estresse o que ela sentia não era fome física, da ordem da necessidade, mas uma fome "pulsional", da ordem do desejo, ligada a sentimentos outrora inacessíveis, neste caso, medo e tensão.

Para McDougall (2000), alguns pacientes vivem como insuportáveis determinados afetos, o que os faz tentar a todo custo dispersá-los imediatamente por meio de uma ação. Podemos pensar que enquanto Antonia mantinha uma relação compulsiva com a comida, estava funcionando nesse registro. Quando ela estava em dieta, ou seja, distante do uso compulsivo da comida, pôde, na continuidade do tratamento e aos poucos, entrar em contato com os afetos que antes eram descarregados no corpo e, com a ajuda da psicoterapia, transformá-los em palavras.

No depoimento a seguir, retirado da página da internet de uma clínica de tratamento da obesidade, Sylvia relata um processo no qual também podemos observar o delineamento de um caminho simbólico onde antes só era possível a expressão somática direta:

Durante uma discussão fiquei muito, muito triste e comecei a sentir fome. Fome? Quanto mais triste mais fome. Conversei dessa vez com a fome e percebi que se fosse comer não ia parar. Estava chegando em casa. Um perigo. Sai de novo e (...) não comi, não continuei a brigar, não contei a briga pra mais 


\section{ARTIGOS}

ninguém. (...) Botei meu problema em perspectiva. Não era tão horrivel assim. Também não era fome. Era tristeza, uma sensação de rejeição, de inadequação que por algum motivo eu acho insuportável e aprendi a chamar de fome. E por que eu não percebia assim antes? Não dava tempo. Eu tratava logo de comer (...) Ao contrário do que eu pensava, (...) Ceder ao impulso de comer no máximo anestesia aquela dor, mas não resolve nada! Só engorda! ${ }^{2}$

Com esse relato também podemos pensar que a retirada do alimento, associada ao processo psicoterapêutico, possibilitou o acesso a angústias que puderam ser vistas e postas em palavras. Os dois casos descritos sugerem situações nas quais as pacientes estavam dispostas a enveredar por um universo desconhecido e assustador, aceitando não só se afastar do objeto-droga, mas se deparar com os aspectos psíquicos subjacentes ao seu uso. Concordamos com McDougall (2000) quando ela diz que "o tratamento pela psicanálise só é potencialmente eficaz se o paciente quiser realmente descobrir por que recorre ao objeto adictivo ao menor sinal de stress" (p. 110).

No entanto, como dissemos anteriormente, também foi possível observarmos em nossa prática clínica pacientes que, diante do afastamento do alimento, não tiveram recursos psíquicos suficientes para fazer essa passagem para a nomeação e a elaboração de afetos.

Em alguns casos observamos reações que revelam um mecanismo de defesa que geralmente encontramos nos pacientes fronteiriços e nos psicóticos, mas também, como observou Mcdougall (2000), “em indivíduos não atingidos por psicoses, mas caracterizados por uma economia adictiva e por manifestações psicossomáticas" (p. 140; itálico da autora). Nesses casos a abstinência traz à tona um funcionamento extremamente desorganizado que nos remete à função que a compulsão estava tendo para o psiquismo, ou seja, a de uma "resposta somatopsíquica emitida pelo psiquismo em seu esforço para conjurar as angústias que seriam talvez psicóticas caso chegassem à consciência" (p. 68-69).

Em outros casos, o deslocamento da comida para outro(s) objeto(s)-droga impede o contato com a angústia. É o que aconteceu com Tereza (nome fictício), que estava há 6 meses em tratamento e era considerada por todos os outros pacientes um exemplo de rigor na dieta. Emagrecera muito e mostrava estar alegre e confiante por ter reduzido a numeração de suas roupas e por sustentar seus "nãos" diante das comidas que ela adora. Esse "caso modelo",

$2 \mathrm{https}: / / \mathrm{ww}$. facebook.com/ espacoterapeuticopaulobuosi/ photos/a.607543272679359.1073741828.605855806181439/792457410854610/?type=3\&theater 
no entanto, preocupava os terapeutas, uma vez que não conseguiam enxergar nenhum conflito: estava tudo bem demais. Nenhum sofrimento aparente, nenhum novo questionamento diante do "vazio" que a retirada da comida abrira, nenhuma angústia. Até que em um grupo, Tereza, com muita dificuldade e relatando estar muito envergonhada, contou que estava, há alguns meses, comprando compulsivamente e que alcançara o limite do cartão de crédito pela primeira vez na vida. Mesmo isso tendo vindo à tona, não significou a possibilidade de ser trabalhado: sua fala teve mais um caráter confessional do que de abertura para elaboração. Tereza encerrou-se novamente na personagem-modelo até atingir o peso sonhado e abandonou o tratamento. Cerca de um ano depois, um encontro casual nos colocou no mesmo ambiente: ela havia engordado praticamente tudo o que perdera na clínica. Contou, na ocasião desse encontro, que alguns meses depois de ter saído da clínica foi aos poucos voltando a comer tanto quanto ou mais do que comia antes.

\section{Considerações finais}

Sublinhamos que a abordagem teórica que orienta a clínica na qual a experiência profissional ocorreu considera a abstinência do objeto-droga, ou seja, da comida, fundamental para o tratamento. Embora a psicanálise não seja proscrita do olhar desta clínica, ela ocupa um lugar menor tanto nos grupos de psicoterapia quanto, como vimos, nas publicações sobre a obesidade. Vejamos como Ravenna (2006), importante referência teórica na clínica na qual o trabalho foi realizado, vê a questão da psicoterapia:

(...) embora a Psicanálise não seja a mais adequada para o tratamento da obesidade, podemos, contudo, extrair daí algumas descrições muito úteis para análise da personalidade do obeso, suas tendências aditivas e os mecanismos de defesa que desenvolve (...). Embora cada situação particular exija indubitavelmente uma abordagem terapêutica determinada, com o passar dos anos descobrimos que, no tratamento da obesidade, a perda rápida de peso e a consequente mudança de hábitos são obtidas fundamentalmente com a implementação de terapias derivadas do Comportamentismo ou Behaviorismo, como método baseado antes no empirismo e não na interpretação subjetiva, ou seja, centrado nos comportamentos observáveis e não nas motivações inconscientes. ( $\mathrm{s} / \mathrm{p}$; itálicos nossos) 


\section{ARTIGOS}

Entendemos que a psicanálise pode oferecer mais do que Ravenna sugere no trecho acima. $O$ percurso do presente texto sugere a contribuição da psicanálise para o enfrentamento de pelo menos dois problemas e limites que observamos nesta prática: o tratamento da compulsão alimentar sem levar em consideração as diferenças de constituição subjetivas e a dificuldade na manutenção do peso baixo por longo tempo.

No primeiro caso, este estudo alerta para o risco de graves desorganizações psíquicas de alguns pacientes quando submetidos à dieta de alta restrição calórica. Quanto ao segundo caso, entendemos que os ciclos de perda e ganho recorrentes são um indicativo importante de que não houve um trabalho psíquico concomitante ao processo de emagrecimento. Entendemos que se a compulsão alimentar pode ser barrada e contornada, de modo efetivo, por algumas perspectivas de tratamento para o emagrecimento na atualidade, frequentemente o peso retorna depois do final do tratamento, justamente pela impossibilidade de colocar a problemática da compulsão alimentar em trabalho psíquico.

Uma abordagem psicanalítica da compulsão alimentar, assim, se contrapõe ao imperativo social do emagrecimento, como melhor solução para todos os indivíduos, convocando-nos a reconhecer que para cada sujeito e em cada situação, há ganhos e perdas diferentes a serem considerados. Paralelamente, uma abordagem psicanalítica da questão sublinha a possibilidade e necessidade, para alguns sujeitos, de um tratamento das angústias psíquicas tamponadas pela compulsão alimentar, como caminho para uma relação mais saudável com a comida e com a vida.

\section{Referências}

American Psychiatric Association. (2014). DSM-5: Manual diagnóstico e estatístico de transtornos mentais (5a ed.). Porto Alegre, RS: Artmed.

Castanho, P. (2014). Sobre como trabalha um analista ao coordenar um grupo. Vinculo, 11(2), 41-52.

Espíndola, C. R., \& Blay, S. L. (2006). Bulimia e transtorno da compulsão alimentar periódica: revisão sistemática e metassíntese. Revista de Psiquiatria do RS, 28(3), 265-75. doi: 10.1590/S0101-81082006000300006.

Fernandes, M. H. (2011). Corpo (4a ed.). Coleção Clínica Psicanalítica. São Paulo, SP: Casa do Psicólogo.

Ferraz, F. C. (2011). A tortuosa trajetória do corpo na psicanálise. In F. C. Ferraz, Ensaios Psicanalíticos (pp. 237-259). São Paulo, SP: Casa do Psicólogo. 
Gurfinkel, D. (2011). Adicções. Coleção Clínica Psicanalítica. São Paulo, SP: Casa do Psicólogo.

Jeammet, P. (2008). A abordagem psicanalítica dos transtornos das condutas alimentares. In R. Urribarri (Org.), Anorexia e bulimia (pp. 20-49). São Paulo, SP: Escuta.

Kalil, F. (2010). A compulsão alimentar e suas implicações na clínica psicanalítica. In A. P. Gonzaga, \& C. Weinberg (Orgs.), Psicanálise de transtornos alimentares (pp. 167-185). São Paulo, SP: Primavera Editorial.

Klein M. (1991). Notas sobre alguns mecanismos esquizoides. In M. Klein, Inveja e gratidão, e outros trabalhos (1946-1963) (pp. 20-43). Rio de Janeiro, RJ: Imago. (Trabalho original publicado em 1946).

Magdaleno, J. R., Chaim, E. A., \& Turato, E. R. (2009). Características psicológicas de pacientes submetidos à cirurgia bariátrica. Revista de Psiquiatria do Rio Grande do Sul, 31(1), 73-78. doi: 10.1590/S0101-81082009000100013.

Marcelino, L. F., \& Patrício, Z. M. (2011). A complexidade da obesidade e o processo de viver após a cirurgia bariátrica: uma questão de saúde coletiva. Ciência \& Saúde Coletiva, 16(12), 4767-4776. doi: 10.1590/S1413-81232011001300025.

McDougall, J. (1983). Em defesa de uma certa anormalidade: teoria e clínica psicanalítica. Porto Alegre, RS: Artes Médicas.

McDougall, J. (1995). As múltiplas faces de Eros. Uma exploração psicoanalítica da sexualidade humana (P. H. B. Rondon, trad.). São Paulo, SP: Martins Fontes.

McDougall, J. (2000). Teatros do corpo: o psicossoma em psicanálise (2a ed.). São Paulo, SP: Martins Fontes.

Mundim, E. R. (1996, jan./mar.). Obesidade: Reflexões. Revista Médica de Minas Gerais. 6(1), 20-25.

Ravenna, M. (2006). A teia de aranha alimentar. Quem come quem? (E. Aguiar, trad.). Rio de Janeiro, RJ: Guarda-Chuva.

Tavares, T. B., Nunes, S. M., \& Santos, M. D. O. (2010). Obesidade e qualidade de vida: revisão da literatura. Revista de Medicina Minas Gerais, 20(3): 359-366. Recuperado de: <http://rmmg.org/artigo/detalhes/371>.

World Health Organization (2015). Fact sheet: obesity and overweight. Recuperado de: <http://www.who.int/mediacentre/factsheets/fs311/en/>.

Wulff, M. (2003). Sobre um interessante complexo sintomático oral. In B. Brusset, C. Couvreur, \& A. Fine, A bulimia (pp. 59-79). São Paulo, SP: Escuta. (Trabalho original publicado em 1932).

Zukerfeld, R. (1979). Psicoterapia de la obesidad. Buenos Aires, Argentina: Letra Viva. Zukerfeld, R. (1996). Acto bultmico, cuerpo y tercera tópica. (2a ed.). Buenos Aires, Argentina: Paidós. 


\section{ARTIGOS}

Agradecimentos: Os autores agradecem Marc Kostag, Josiane Bartz e Juan Diego Taborda Lopez pelas cuidadosas traduções para o alemão e para o espanhol e aos membros da oficina de artigos do CLIGIAP pelas ricas leituras e discussões: Cecília de Britto, Daina Bittar, Danna De Luccia, Felipe Martins, Lumi Shine, Marcus Vinicius Barbiero, Marina Cohen, Pedro Carvalho e Sofia Souza.

\section{Resumos}

(Psychic dimensions of weight loss: For a psychoanalytic understanding of binge eating disorder)

This article proposes a theoretical-clinical research with the objective of presenting a psychoanalytic understanding of binge eating disorder. For that sake, we present a critical review of the literature articulated with patients' vignettes and public statements. We conclude by emphasizing the potential of a psychoanalytic approach to binge eating disorder in highlighting the existence of risks of psychic disorganization of some patients undergoing high calorie restriction diets and their potential to deal with the vicious cycle of loss and subsequent weight gain.

Keywords: Eating disorders, obesity, psychoanalysis, compulsive behavior

(Dimensions psychiques d'amaigrissement: Pour une compréhension psychanalytique de la compulsion alimentaire)

Cet article propose une recherche théorique-clinique dans le but de présenter une compréhension psychanalytique de la compulsion alimentaire. Pour tel, nous présentons une revue critique de la littérature articulée par des vignettes et des témoignages publics de patients. Nous conclurons en mettant en relief le potentiel d'une approche psychanalytique de la compulsion alimentaire en mettant en évidence l'existence d'un risque de désorganisation psychique de certains patients subissant des régimes de grand restriction calorique et son potentiel pour faire face au cercle vicieux de la perte et du gain de poids subséquents.

Mots clés: Troubles de l'alimentation, obésité, psychanalyse, comportement compulsif

(Dimensiones psíquicas del adelgazamiento: por una comprensión psicoanalítica de la alimentación compulsiva)

Este artículo propone una búsqueda teórico-clínica con el objetivo de presentar una comprensión psicoanalítica de la alimentación compulsiva. Para hacerlo, presentamos una revisión literaria crítica articulada con viñetas de anotaciones de sesiones terapéuticas y testimonios públicos de pacientes. Concluiremos resaltando el potencial de un abordaje psicoanalítico de la alimentación compulsiva que destaca la 
existencia de un riesgo de desorganización psíquica de algunos pacientes sometidos a dietas de alta restricción calórica, y su capacidad para lidiar con los círculos viciosos de la pérdida y posterior aumento de peso.

Palabras clave: Trastornos de la ingestión de alimentos, obesidad, psicoanálisis, conducta compulsiva

(Psychische Dimensionen der Gewichtsreduktion: Für ein psychoanalytisches Verständnis des Esszwangs)

Dieser Artikel beschreibt eine klinisch-theoretische Studie, die zum Ziel hat, das psychoanalytisches Verständnis des Esszwangs zu vertiefen. Wir beginnen mit einem kritischen Überblick der wissenschaftlichen Literatur und stellen Verbindungen her zu Fallvignetten und öffentlichen Aussagen von Patienten. Daraus schlussfolgert, dass ein psychoanalytischer Ansatz zur Untersuchung des Esszwangs es ermöglicht, eine bestehende oder mögliche psychische Erkrankung von Patienten zu erkennen, die eine stark kalorienbeschränkende Diät befolgen. Dieser Ansatz könnte außerdem den Patienten helfen, die endlose Folge des Ab- und Zunehmens anzugehen.

Schlüsselwörter: Essstörung, Fettleibigkeit, Psychoanalyse, Suchtverhalten

Citação/Citation: Farah, J. F. S., \& Castanho, P. (2018, março). Dimensões psíquicas do emagrecimento: por uma compreensão psicanalítica da compulsão alimentar. Revista Latinoamericana de Psicopatologia Fundamental, 21(1), 41-57. http://dx.doi.org/10.1590/ 1415-4714.2017v21n1p41-4.

Editores do artigo/Editors: Profa. Dra. Ana Maria Rudge e Profa. Dra. Sonia Leite.

Recebido/Received: 18.10.2017/ 10.18.2017 Aceito/Accepted: 5.12.2017 / 12.1.2017

Copyright: (C) 2009 Associação Universitária de Pesquisa em Psicopatologia Fundamental/ University Association for Research in Fundamental Psychopathology. Este é um artigo de livre acesso, que permite uso irrestrito, distribuição e reprodução em qualquer meio, desde que o autor e a fonte sejam citados / This is an open-access article, which permits unrestricted use, distribution, and reproduction in any medium, provided the original authors and sources are credited.

Financiamento/Funding: Os autores declaram não terem sido financiados ou apoiados / The authors have no support or funding to report. 


\section{ARTIGOS}

Conflito de interesses/Conflict of interest: Os autores declaram que não há conflito de interesses / The authors have no conflict of interest to declare.

\section{Juliana Ferreira Santos Farah}

Psicóloga e Psicanalista; Mestranda do Departamento de Psicologia Clínica do Instituto de Psicologia da Universidade de São Paulo - USP (São Paulo, SP, Br); Especialista em Terapia de Família e Casal pela Pontifícia Universidade Católica - PUC-SP (São Paulo, $\mathrm{SP}, \mathrm{Br}$ ).

Rua Capote Valente, 1368

05409-003 São Paulo, SP, Br

julianafarah@usp.br

\section{Pablo Castanho}

Professor Doutor do Departamento de Psicologia Clínica do Instituto de Psicologia da Universidade de São Paulo - USP (São Paulo, SP, Br); Membro do Núcleo de Estudos em Saúde Mental e Psicanálise das Configurações Vinculares - NESME (São Paulo, SP, Br); Membro da Reseaux Groupe et Liens Intersubjectifs e da International Association for Group Psychotherapy and Group Processes (IAGP).

Instituto de Psicologia da USP-PSC

Av. Prof. Mello Moraes 1721 - Bloco F - Cidade Universitária

05508-030 São Paulo, SP, Br

pablo.castanho@usp.br

This is an open-access article, which permits unrestricted use, distribution, and reproduction in any medium for non-commercial purposes provided the original authors and sources are credited. 